\title{
The Direct Preparation of Cigarette Smoke Condensate by High Velocity Impaction*
}

by H.D. Mathewson

Cigarette Components Ltd., London

INTRODUCTION

In recent years, a number of devices and procedures have been described for the collection of whole tobacco smoke, and its constituent phases (e. g. I to 7). Traps for the collection of particulate matter [e. g. Cambridge filter (1), electrostatic precipitator (2)], vapour phase [e. g. solvent scrubbers (3), cold traps (4)], and whole smoke [generally some form of cold trap (4)], are now in use, and each of these methods of collection, as compared with the others, has advantages and disadvantages depending on such factors as the size of smoke sample required, and the subsequent fate of the smoke condensate so produced. The method described in this paper has such acceptable features as simplicity in design, and versatility in its applications, and may be considered to complement, in a number of ways, other collection procedures in current use. This method, which is based on the well-known phenomenon, in aerosol physics, of "condensation by impaction", was developed largely by empirical means, but the theoretical aspects are of interest, and in the next section, these are discussed, briefly and qualitatively, as a general background to the subject matter.

If aerosol particles in a gas stream develop sufficient momentum, they may, on approaching a solid obstruction, cross the streamlines, impinge, and condense on the surface of this obstruction. This principle has been applied in a number of pieces of apparatus used in aerosol sample collection, the cascade impactor (8a) being an example of its application in particle size sampling. A number of factors which determine the efficiency of collection in impaction devices are of importance, and these are now considered in turn.

(a) Conditions of Flow

The streamline flow characteristics of a gaseous system through a constant diameter tube are given by Reynold's Number (Re) which defines the ratio of inertial to viscous forces in the system for a given set of flow conditions. Thus, for a low value of Reynold's Number the flow pattern tends to be controlled primarily by fluid viscosity, and disturbances due to obstacles tend to be-marked well upstream. The gradual displacement of streamlines therefore tends to assist in steering aerosol particles past obstacles, and thus in reducing the chances of impingement caused by particle inertia. On the other hand, a high value of Reynold's Number indicates that flow is predominantly inertiacontrolled, so that disturbance in the streamlines only becomes apparent near to the obstacle. This sudden diversion of the fluid-streams tends to encourage particles of sufficient momentum to cross the streamlines and impact on the surface of the obstruction. However, above a certain transition

$$
\text { "Presented at the CORESTA "Smoke Group" meeting, Les Rasses, January } 1966 .
$$


value of Re, turbulent flow sets in, and particle impaction becomes increasingly inefficient. Thus, ideally, to obtain highly efficient collection of aerosols by impaction conditions should be adjusted so that Reynold's Number is as high as is consistent with keeping the flow laminar. This may be achieved by increasing of the mean velocity of the fluid through the tube, or by enlarging the bore of the tube, these two factors being interrelated through the flow rate, which, for most systems, must lie within certain limits.

(b) Aerosol Droplet Size

The momenta of the aerosol particles depend, of course, upon their mass, and as one proceeds farther down the size scale the velocity required to drag the droplet from the carrier gas streamlines becomes greater. As the velocity of the stream is decreased, therefore, the very smallest particles are the first to escape impact, an increasing proportion of larger droplets being carried through as the velocity drops further. Also, at higher velocities, the smaller diameter of the drop, the less chance there is of "interception" by contacts made other than by direct impingement. This factor becomes important in multi-unit collectors such as aerosol filters, although impaction processes are considered to be of secondary importance in such filters.

(c) Adhesion to the Surface

Whether or not contact between a rapidly moving aerosol particle and a fixed surface produces adhesion depends upon a number of factors. The main parameters concerned are the size and velocity of the particles, and their chemical nature, the physical and chemical properties of the surface, and the area of contact between droplet and surface on impact. For inertial impaction, the directness of the particle-surface collision, the "stickiness" of the aerosol material, and the physical nature of the surface have the greatest influence on collection efficiency. The adhesion of droplets to the surface of impingement, and hence the efficiency of collection is often increased once a layer of liquid condensate has been formed.

(d) Electrical Effects

Effects due to surface electrostatic charge probably come into play only where the aerosol mean velocity is sufficiently low for Brownian diffusion and sedimentation phenomena to occur. Electrical interactions are likely to play a subsidiary role in impaction systems.

In the tobacco smoke traps which are in current use, condensation due to impaction does not occur to a large extent because of the relatively small size of most of the aerosol population [0.1 $-1.0 \mu$ diameter (9)], combined with the comparatively low mean velocity in most collection devices. The aerosol velocity may be increased by decrease in the diameter of the flow system, but the only "nozzle-type" impactor to have been so far described in the literature is the "pressure-smoking apparatus" of Neurath and Kröger (7), where impaction undoubtedly contributes to some degree in the production of condensate. Other "capillary" traps such as that of Cuzin (5), and the more recent "capillary press" collectors of Seehofer and Hanßen (6) are based on "forced coagulation" brought about by turbulent flow inside narrow-bore capillary tubes, and conditions are sufficiently different such that these traps do not bear direct comparison with the device which is described in the next section.

\section{NOZZLE IMPACTION}

In nozzle-type impactors, the aerosol is passed through an orifice at high speed, and allowed to impact on to a flat surface a short distance away. If the factors mentioned above are favourable (high Reynold's Number, good adhesion, etc.), a high efficiency of collection may be obtained by such a unit, the aerosol size range collected depending on the dimensions of the impaction area, and on the flow rate of the aerosol stream. For tobacco smoke collection systems, the dimensions of the apparatus are, of course, strictly limited by the smoking parameters, unless, as in the capillary press, the condensation is produced by a secondary process. In the apparatus described here, the trapping is direct, and it is of interest to compare the best conditions arrived at empirically with those expected from theoretical considerations.

To produce a $35 \mathrm{ml}$. puff of 2 seconds duration, a flow rate of $17.5 \mathrm{ml} . / \mathrm{sec}$. is required for the smoke 
FIGURE]

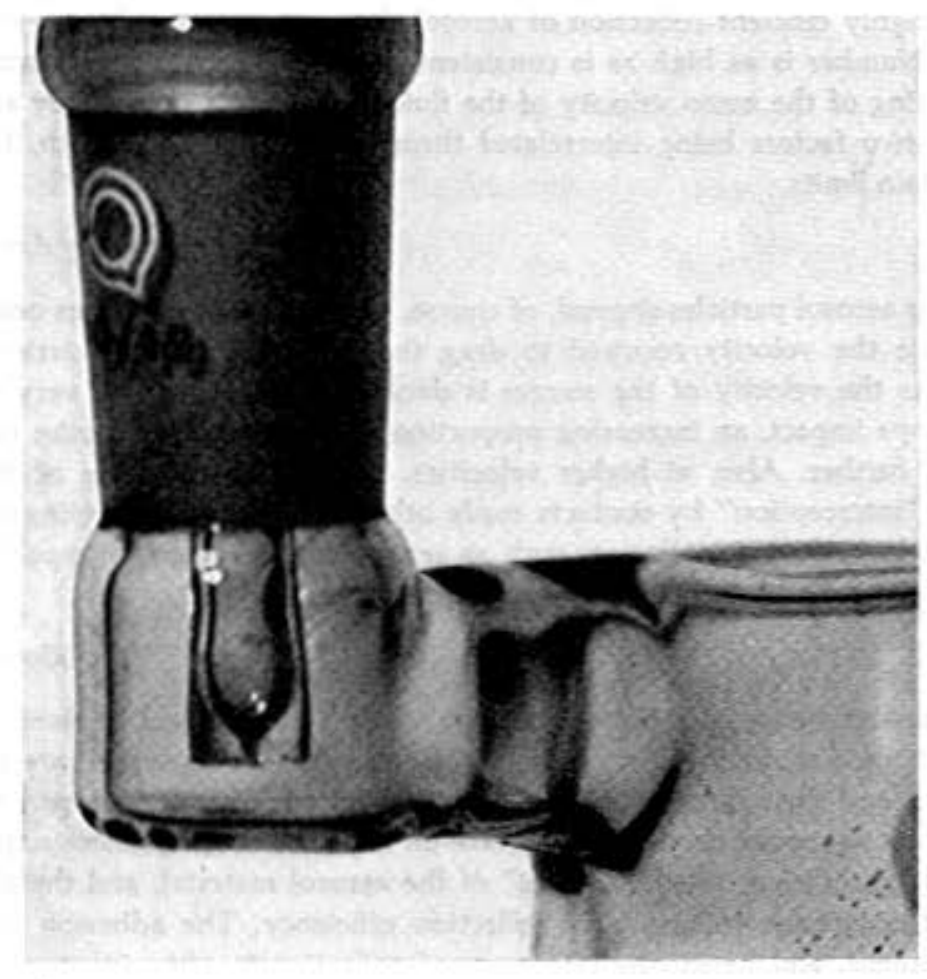

FIGURE?

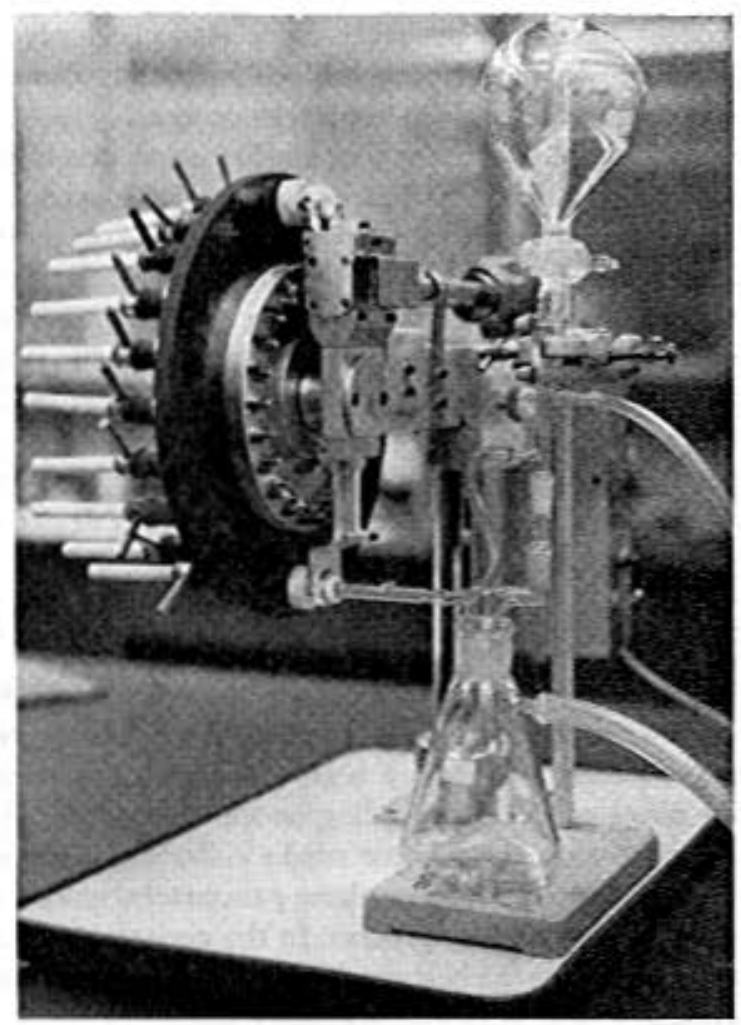


emerging from the cigarette. A jet diameter of $0.4 \mathrm{~mm}$. at a distance of $1-2 \mathrm{~mm}$. from the impaction surface, and a downstream pressure of $190 \mathrm{~mm}$. Hg. below atmospheric were found to satisfy the smoking parameters, whilst allowing the passage of less than $2 \%$ of the total particulate matter in the smoke. A close-up of the impaction area of the all-glass mass impaction trap is shown in Figure 1. For the above conditions, Reynold's Number for air has been calculated as 3700 , a figure which brings the flow well into the region of turbulence*. However, if the jet diameter is enlarged, so that for the same flow rate, turbulence is reduced, the efficiency of collection of condensate is lowered. This is partly explained by the small average size of the smoke aerosol particles, a high velocity being required to give them sufficient momentum to cross the flow-lines. Also the high collection efficiency for such high calculated $R e$ values is better understood if the flow pattern through an orifice rather than a constant diameter tube is considered. Turbulent flow through orifices has been examined in detail with respect to flow-metering devices such as the Venturi Tube (9). The main conclusions are that when a gas flows through a tube which narrows suddenly to an orifice, the emergent high velocity stream initially follows the shape of a narrowing cone, the cross-sectional area being at a minimum, the velocity at a maximum at a point known as the vena contracta. Beyond this point the main gas stream flares out rapidly. This central high-velocity region is surrounded by a region of turbulence. It is believed that this central flow region, which will contain most of the aerosol particles, is chiefly responsible for the efficient impaction and condensation, and the experimental evidence that impaction efficiency increases rapidly as the impact surface approaches the jet exit is in accord with theory, the vena contracta lying within the first millimetre or so of the emergent jet of smoke. Within $2 \mathrm{~mm}$. the position of the surface does not appear to effect the efficiency further.

Since most of the development work has been concerned with producing a trap capable of collecting smoke particulate matter in bulk quantities, this version, the mass impaction trap, is described in the greatest detail.

THE MASS IMPACTION TRAP

The impaction area shown in Figure $I$, is part of the mass condensate trap, a photograph of which is shown in Figure 2. It can be seen that the trap is mounted behind the fixed inlet port of a 24 port rotary smoking machine. The components of the trap are labelled in Figure 3. The pneumatics are arranged so that there is a permanent reduced pressure downstream of the nozzle (ca. $190 \mathrm{~mm}$. $\mathrm{Hg}$ ), smoke being drawn through the orifice during the twosecond interval that the lit cigarette is in front of the stationary port, while the bypass is shut off by means of a timed electromagnetic valve. During the half-second interval between puffs, air is drawn in through the bypass inlet while the smoking inlet is closed off by the rotating disc.

Owing to the eventual accumulation of condensate in the impaction area, after the smoking of large numbers of cigarettes, it was found expedient to incorporate a solvent reservoir in the trap, acetone being chosen for the present system. As can be seen from the diagrams, solvent can be admitted at any time without interrupting smoking. The pneumatic circuit including flow-monitoring apparatus, is shown in Figure 4.

\footnotetext{
- Turbulence sets in for $\overline{\operatorname{Re}>2300-2400 \text {. }}$
}

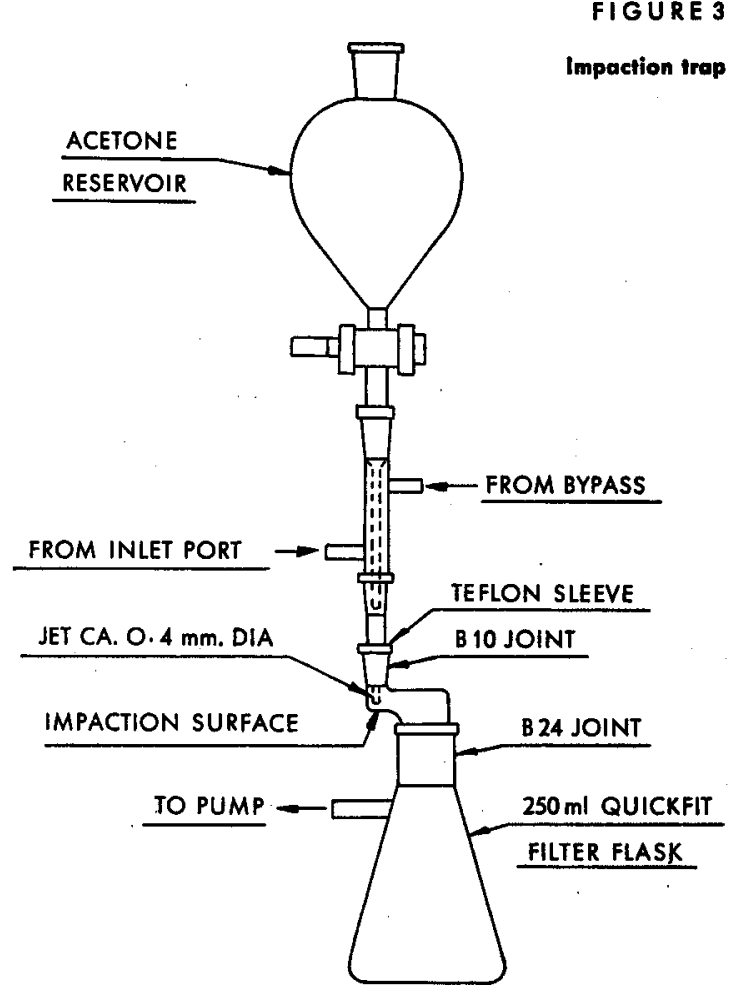




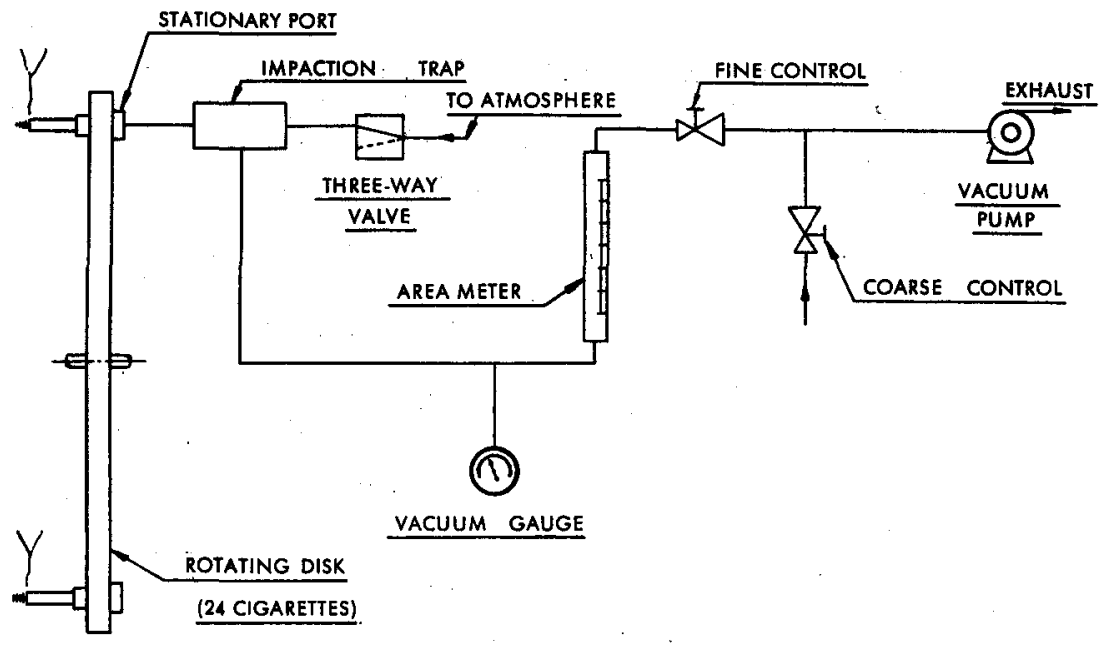

Because of the high pressure drop of the trap, the emergent vapour phase is subjected to changes in viscosity and volume which prevent direct measurement of flow rates. It was therefore found necessary to introduce a vacuum gauge downstream of the impactor, a calibration line of vacuum against area meter reading for variations in pressure drop being plotted for a fixed upstream flow rate of $17.5 \mathrm{ml} /$ second. The puff volume for a given set of smoking conditions can then be determined exactly using a needle valve in place of the impaction trap.

To ensure all smoking parameters were satisfactory, the puff profile was measured using a kymograph attached immediately downstream of a cigarette. A typical result is shown in Figure 5, where the shape is seen to be satisfactorily rectangular. The dip in the curve at the end of the puff is due to the inertia of the pen on its rapid descending stroke.

FIGURE 5

Puff profle

Kymograph record of pressure changes downstream of cigarette during puff
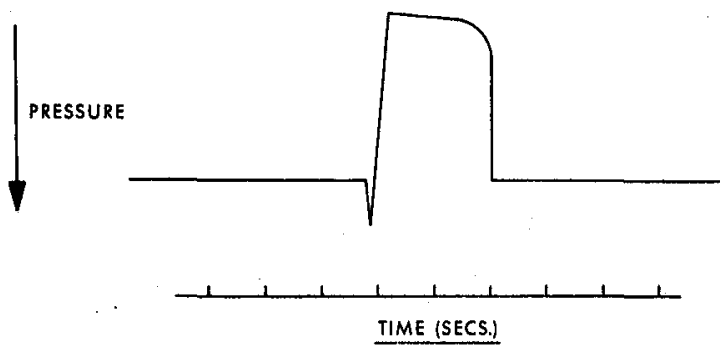

Analysis of the Condensate

The yields of condensate, condensate moisture*, and nicotine** were determined for the mass impactor, and these are shown in the table in Figure 6, along with the equivalent figures for tar collected on a Cambridge filter. It is seen that the values for moisture and dry tar are somewhat lower for the impaction trap than for the Cambridge filter. These results are plausible, since the fibre-glass filter is known to become a more efficient filter for certain volatile smoke constituents when there is a build-up of collected condensate. This solution effect would be considerably lessened for the impactor, where the area of contact between vapour phase and condensate is very much lower. In addition, the reduced pressure, and intermittent "clearing puffs" of air inevitably encourage volatilisation of lower-boiling constituents in the impactor condensate. The differences in the content of "semi-volatiles" in the two forms of condensate are seen clearly in the gas-liquid chromatograms shown in Figure 7 , the samples being processed according to the distillation/chromatography procedure described by Williamson, Graham and Allman (1I). The samples differ only in a quantitative sense, the compositions approaching one another and becoming identical in the content of less volatile compounds. In this sense, nico-

FIGURE 6

\begin{tabular}{l|ccc|c|c}
\hline $\begin{array}{l}\text { Method of } \\
\text { collection }\end{array}$ & $\begin{array}{c}\text { Wet tar } \\
\text { produced } \\
\text { (mg./cig.) }\end{array}$ & $\begin{array}{c}\text { Dry tar } \\
\text { produced } \\
\text { (mg./cig.) }\end{array}$ & $\begin{array}{c}\text { Moisture } \\
\text { content } \\
\text { (mg./cig.) }\end{array}$ & $\begin{array}{c}\text { Moisture } \\
\text { in tar } \\
(\%)\end{array}$ & $\begin{array}{c}\text { Nicotine } \\
\text { produced } \\
\text { (mg./cig.) }\end{array}$ \\
\hline Cambridge filter & 46 & 36.5 & 9.5 & 21 & 2.3 \\
Impaction trap & 41 & 34 & 7.0 & 17 & 2.3 \\
\hline
\end{tabular}

Smoking conditions :

$55 \mathrm{~mm}$. Virginia blend cigarettes smoked to a $9 \mathrm{~mm}$. butt length. Puff volume: 35 c.c.

Average number of puffs: 9. tine acts as a "non-volatile", the average nicotine production being $2.3 \mathrm{mg}$./cigarette for both impactor and Cambridge filter. The gas chromatograms confirm that there are no losses of nicotine in the impaction trap.

* Karl Fischer titration. ** Modified Willit's method (10). 

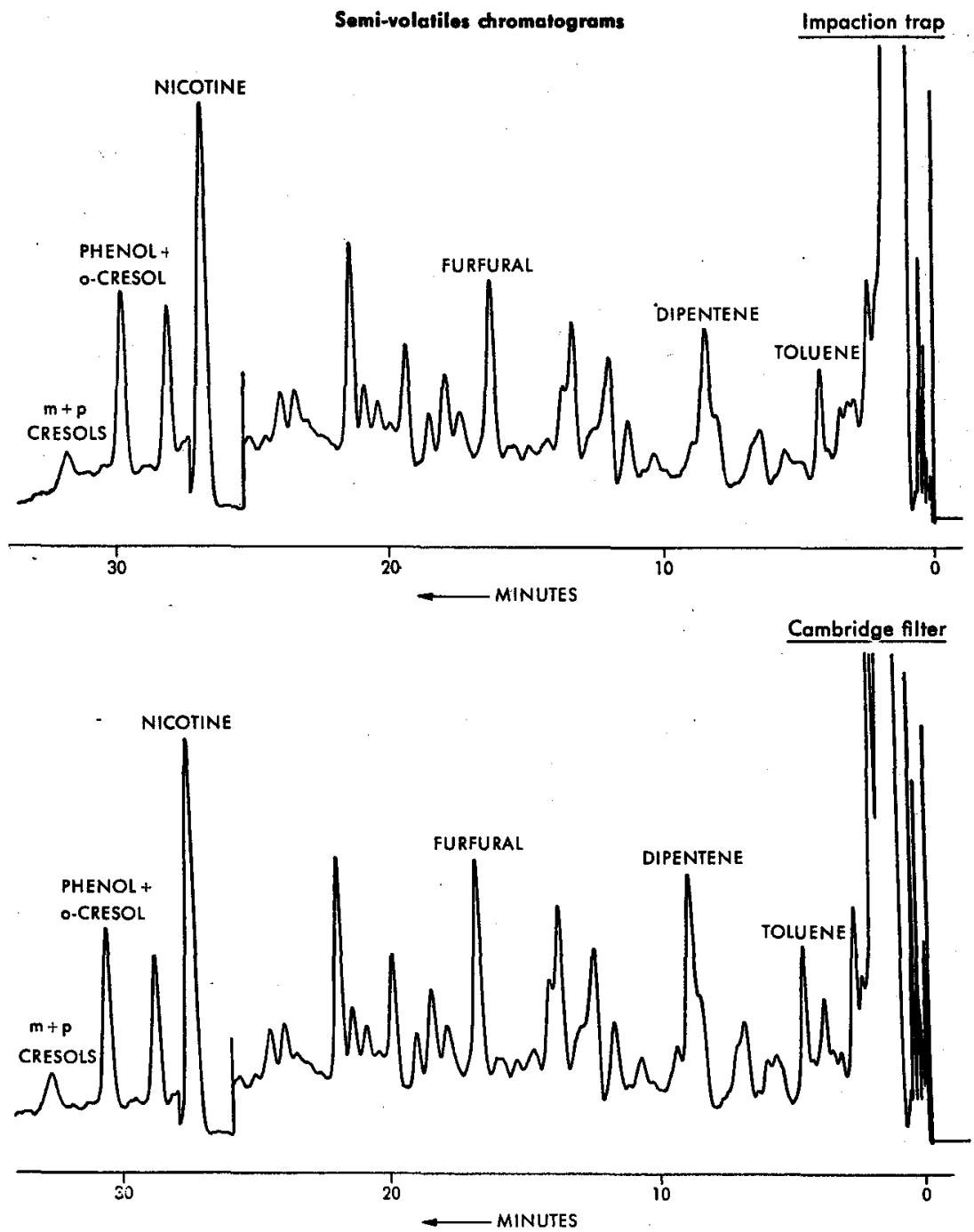

Where concentrated condensate solutions are required (stronger than $50 \% \mathrm{w} / \mathrm{v}$ ), it is necessary to add small frequent doses of acetone through the flushing system. To reduce operator manipulations, it has been found possible to add solvent aliquots automatically by means of a repeating dispenser coupled mechanically to the rotating head of the smoking machine. One o.I ml. aliquot of acetone added once per revolution of the disc was found sufficient to keep the smoking conditions steady during a number of successive smokings.

The method described above offers a useful means of collecting condensate from many hundreds of cigarettes as a concentrated solution in acetone, the trap being compact in size, simple in design, and easy to operate.

THE ANALYTICAL IMPACTION TRAP

Work is proceeding satisfactorily on a smaller analytical version of the impaction trap, one example of which is shown on the photograph in Figure 8. The trap shown is capable of collecting condensate from up to $\mathbf{7 2}$ cigarettes in the neat condition. It fits into the pneumatic circuit shown in Figure 9. In this diagram, it can be seen that there is a large ballast tank which, in addition to stabilising the flow through the system, is capable of reducing the pressure inside the trap from atmospheric to the operating vacuum in less than $0.2 \mathrm{sec}$. By altering the timing of the coupled electromagnetic valves such that the trap is "switched in" a small fraction of a second before the rotating head is in position for a puff, the full operating pressure difference is applied during each entire puff, and collection efficiency is high. This set-up eliminates the "clear- 
ing puff" which is necessary in the mass

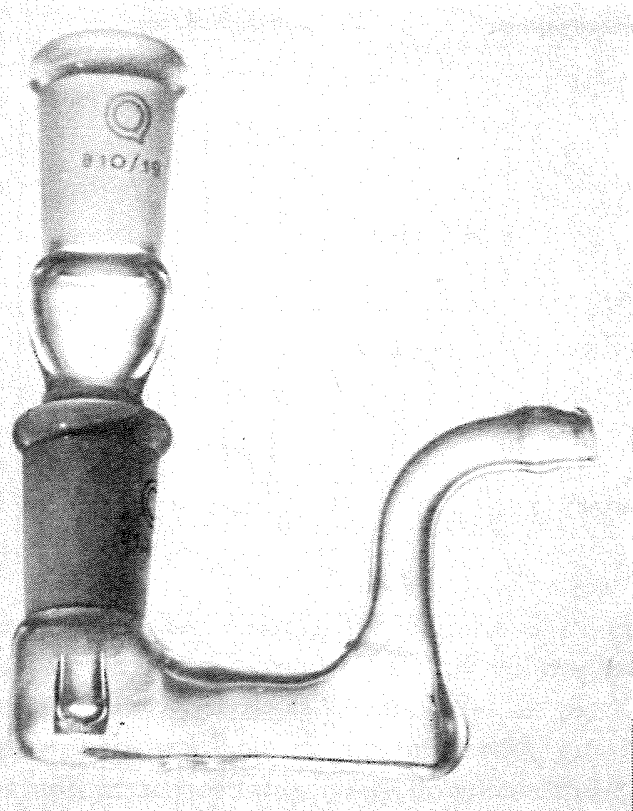

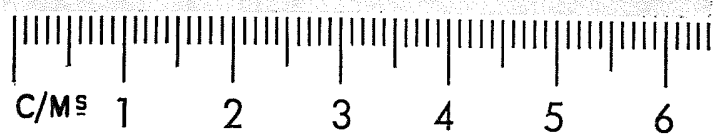
impactor, and reduces the time during which the condensate is exposed to reduced pressure. This impaction trap has the advantages over the Cambridge filter that larger amounts of condensate can be collected and that the condensate produced is in the neat condition, and may be examined directly, or in solutions of any required concentration. The form of impaction traps is by no means limited to the types described above, and the principle may be applied to a large variety of modifications. Some recent experiments indicate that one such modification, which is less susceptible to clogging by deposited condensate than the models already described, may extend considerably the amount of tar which may be collected in a mass unit without the need for a solvent flushing system.

\section{APPLICATIONS OF IMPACTION COLLECTORS}

In addition to replacing or complementing existing trapping methods, impaction traps may be used in some applications for which other

collectors are unsuitable. It is well known, for example, that the trapping efficiency for impactors depends on the aerosol particle size, for reasons already discussed. Thus, by using nozzles of different diameters, and at different distances from the impaction surface, particle-size fractions could be collected and analysed. This offers a means of tackling the as yet unresolved problem of how aerosol composition varies among droplets of different sizes. However, a number of problems are encountered, associated with the high concentration of aerosol particles in undiluted smoke, and with the inevitable accumulation of condensate in all forms of impactor so far examined. Methods for eliminating these problems are being examined.

Impactors could also be used to study the equilibrium of aerosol and vapour phase under various conditions, especially if some mechanical device were used to remove the condensate as soon

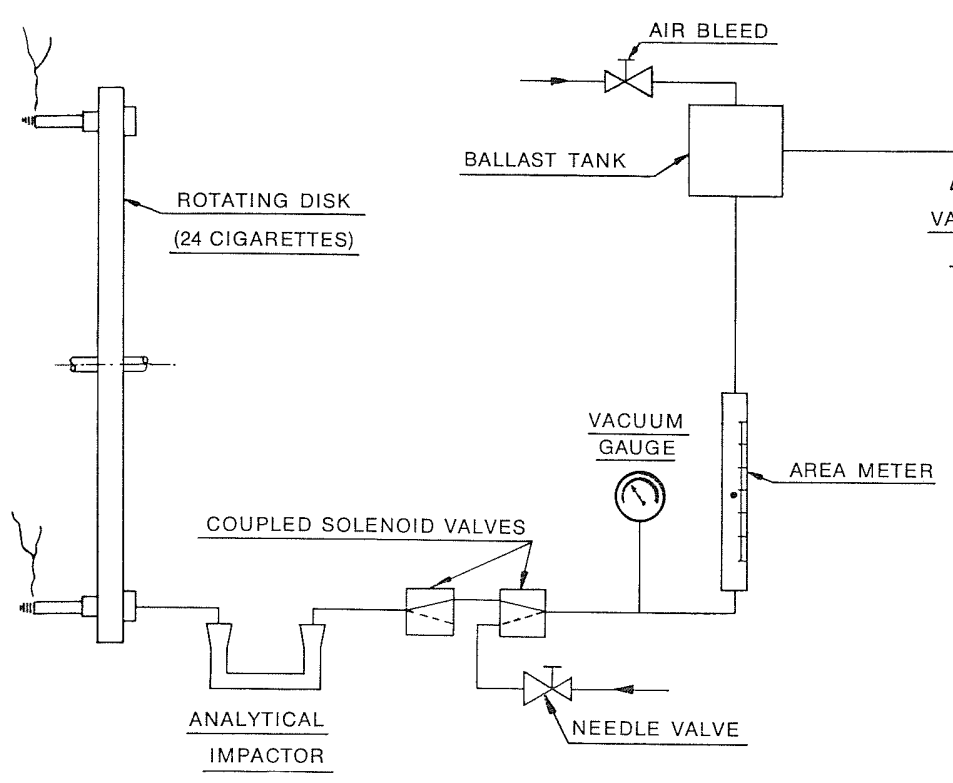

as it is formed. A number of such methods are under consideration.

By placing an impactor in series with an empty U-tube which is immersed in liquid oxygen, an efficient whole-smoke trap is produced. This form of trap has the chief advantage over other cold traps that the particulate phase, the most difficult to re- 
move efficiently, is already dealt with, so that only the vapour phase needs to be condensed out. The result is a much more compact unit than the usual whole-smoke collection apparatus. It is expected that the versatility of impaction traps will be extended considerably by employing the "capillary press" principle developed by Seehofer (7).

SUMMARY

A method is described for the collection of smoke condensate by high-velocity impaction. Analytical and mass-condensate versions are considered, and the results of analyses on their product are reported and discussed. The potential scope of applications of this type of collector are also indicated.

ZUSAMMENFASSUNG

Eine Methode zur Gewinnung von Rauchkondensat durch Aufprall bei hoher Geschwindigkeit wird beschrieben. Die Anwendung für analytische Arbeiten und für die Gewinnung großer Mengen von Kondensat wird erörtert. Analysenergebnisse werden mitgeteilt und diskutiert. Die möglichen Arten der Anwendung dieses Niederschlagsverfahrens werden aufgezeigt.

RESUME

Une méthode de préparation directe de condensat de fumée de cigarettes par impaction à grande vitesse est décrite. Le piège à impaction se prête à la production de grandes quantités de condensat ainsi qu'au fumage analytique. Les résultats d'expériences effectuées sont indiqués et discutés. Les possibilités diverses de l'application du procédé sont présentées.

\section{REFERENCES}

I. Wartman, W. B., Cogbill, E. C., and Harlow, E. S.: Anal. Chem. 31 (1959) 1705.

2. Golaz, P., Girardet, A., and Regamey, R.: Trav. Chim. Alim. et Hyg. 50 (1959) 18.

3. CORESTA, Smoke Group, Budapest 1962: Proceedings, Appendix 3; Keith, C. H., and Newsome, J. R.: Tobacco Sci. 1 (1957) 51; Elmenhorst, H.: Beitr. Tabakforsch. 3 (1965) 101.

4. Barkemeyer, H., and Seehofer, F.: Beitr. Tabakforsch. I (1961) 93; ibid. 3 (1965) 91.

5. Cuzin, J.: Actes du Deuxième Congrès Scientifique International du Tabac, 1958, Brussels, p. 517.

6. Neurath, G., and Kröger, R.: Beitr. Tabakforsch. 2 (1963) 81.

7. Seehofer, F., and Hanßen, D.: Beitr. Tabakforsch. 2 (1964) 303; ibid. 3 (1965) 291.

8. Green, H. L., and Lane, W. R.: Particulate Clouds - Dusts, Smokes and Mists; E. and F. N. Spon Ltd., London, 1957.

8a. Ditto, pp. 233-237.

9. Keith, C. H., and Derrick, J. C.: J. Coll. Sci. 15 (1960) 340.

10. Redding, T. H.: Flow through Orifices and Parallel-Throated Nozzles, Chapman and Hall Ltd., London, 1952, and bibliography therein.

11. Willits, C. O., Swain, M. L., Connelly, J. A., and Brice, B. A.: Anal. Chem. 22 (1950) 430; Laurene, A. H., and Harrell, T. G.: ibid. 30 (1958) i800.

12. Williamson, J. T., Graham, J. F., and Allman, D. R.: Beitr. Tabakforsch. 3 (1965) 233.

The author's address:

Cigarette Components Ltd., Friendly House, 21-24 Chiswell Street, London E.C. 1, England 